\title{
Mitochondrial Aconitase Binds to the 3'-UTR of Mouse Hepatitis Virus RNA
}

\author{
SANTOSH K. NANDA AND JULIAN L. LEIBOWITZ \\ Department of Pathology and Laboratory Medicine, Texas A\&M University System Health \\ Science Center, College Station, Texas 77843-1114
}

\section{INTRODUCTION}

Cis-acting signals located in the 3'-UTR of the viral genome were first identified by deletion analyses of MHV defective interfering (DI)-RNAs (Kim, Jeong, and Makino, 1993; Lin and Lai, 1993). The cis-acting signals for the synthesis of minus-strand RNA are contained within the last 55 nucleotides (nt) plus the poly (A) tail (Lin, Liao, and Lai, 1994). Our lab has previously shown that host cell proteins specifically bind to two distinct sites within the MHV 3'UTR (Yu and Leibowitz, 1995b). In the current work, we show that the RNA-protein (RNP) complex formed within the last $42 \mathrm{nt}$ of the genomic RNA contains four proteins of molecular mass $90,70,58$ and $40 \mathrm{kDa}$ and identify the $90 \mathrm{kDa}$ protein as mitochondrial aconitase.

\section{MATERIALS AND METHODS}

\subsection{RNase protection/gel mobility shift and UV cross- linking assays}

Cytoplasmic extracts were prepared from Dounce homogenized 17Cl-1 cells by a modification of a previously described method (Yu and Leibowitz, 
1995b). RNase protection/gel mobility shift and UV cross-linking assays were performed as described (Yu and Leibowitz, 1995b). Unlysed cells and nuclei were removed by centrifugation at $750 \mathrm{~g}$ for $10 \mathrm{~min}$. The supernatant was centrifuged at $10,000 \mathrm{~g}$ for $30 \mathrm{~min}$ at $4^{\circ} \mathrm{C}$ and this supernatant was stored as the post-mitochondrial fraction.

\subsection{Purification of RNA binding proteins}

Proteins which interacted with the last 42 nt of the MHV genome [3'(+)42] were purified from cytoplasmic lysates by sequential batch fractionation over High Q and High S ion exchange matrices (Bio-Rad) and a heparin agarose matrix (Sigma). At each step samples were assayed for RNA binding activity and analyzed by SDS-PAGE.

A biotinylated synthetic RNA corresponding to nt $42-5$ at the $3^{\prime}$ end of the MHV genome [position 1 is the first nt upstream of the $3^{\prime}$ poly(A) tail] was purchased from Dharmacon Research. The RNA solution was adjusted to $100 \mathrm{mM} \mathrm{KCl}, 5 \mathrm{mM} \mathrm{MgCl}$ and $1 \mathrm{mM}$ DTT and bound to $1 \mathrm{mg}$ of BioMag Streptavidin beads (PerSeptive Biosystems). The eluate from the heparin agarose matrix was added to the beads for $2 \mathrm{~h}$ at $4^{\circ} \mathrm{C}$. After four washes proteins were eluted with $2 \mathrm{M} \mathrm{KCl}$ or by boiling in SDS-PAGE loading buffer.

\subsection{Peptide sequencing}

The partially purified $90 \mathrm{kDa}$ protein was resolved from other proteins by SDS-PAGE, located by staining, and was cut out from the gel. The gel slice was digested with trypsin and the resulting tryptic peptides purified by HPLC and analyzed by MALDI mass spectrometry. Two peptides were subjected to sequential Edman degradation.

\section{RESULTS}

\subsection{Partial purification of the MHV-JHM 3' (+) RNA binding proteins}

Cytosolic extracts from murine $17 \mathrm{Cl}-1$ cells were assayed with a ${ }^{32} \mathrm{P}$ labeled transcript corresponding to nt 16-84 upstream from the 3' end of the MHV-JHM genome. Three RNA-protein complexes formed, with the 
slowest migrating complex, complex 1, being the most abundant (Figure 1A). The specificity of the RNA-protein binding was confirmed by competition experiments (not shown). Molecular masses of cytoplasmic proteins that bind to the MHV-JHM 3'(+)42 RNA were estimated by UVinduced cross-linking assays. Four proteins of 90, 70, 58 and $40 \mathrm{kDa}$ were consistently detected (Fig. 1B). The $90 \mathrm{kDa}$ protein was the most prominent. In situ cross-linking (not shown) revealed that complex 1 contained the same four protein species.

RNA-binding proteins were purified from cytoplasmic lysate as described in Materials and Methods and assayed by gel mobility shift/RNase protection (Fig. 1A), UV cross-linking, and SDS-PAGE (Fig. 1B). Virtually all of the RNA binding activity was in the High Q matrix flow through fraction. This fraction was bound to High $\mathrm{S}$ matrix and RNA binding activity was then eluted with $150 \mathrm{mM} \mathrm{KCl}$. This fraction was subjected to non-specific affinity chromatography with heparin-agarose. This purification scheme enriched for RNA-binding proteins. A $90 \mathrm{kDa}$ protein present in material purified by this method was well resolved from other proteins and strongly labeled by UV cross-linking (Fig. 1B). Two-dimensional gel electrophoresis of this material indicated that this $90 \mathrm{kDa}$ band was a single protein (not shown).
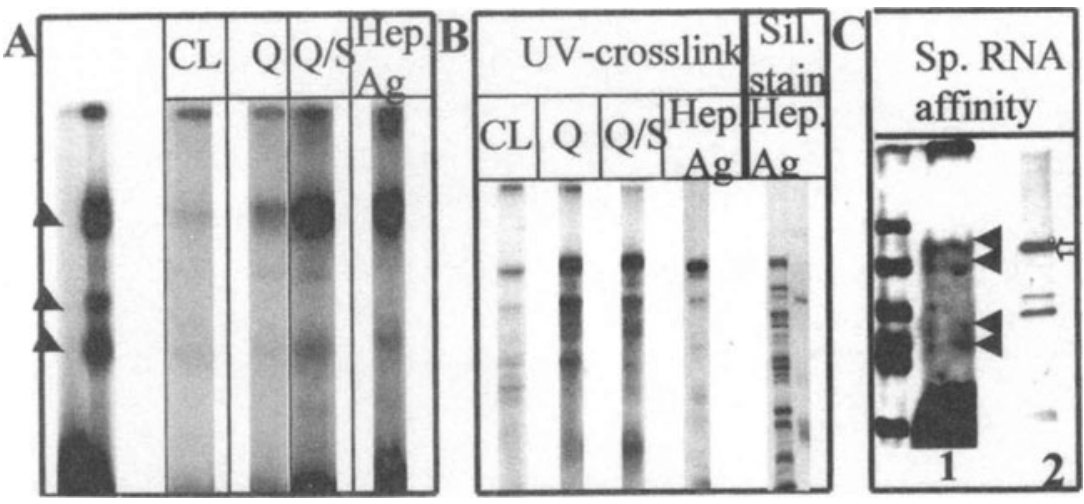

Figure 1. Panel A. A standard RNase protection gel mobility shift assay in on the left. The enrichment of binding activity during purification from crude lysate $(\mathrm{CL})$ and from matrices as described in the text. Panel B. UV cross-linking patterns obtained during purification. Panel C. Affinity purified material visualised by silver staining (lane 1) and by Western Blot with anti-m-aconitase antibody (lane 2). Molecular wt. markers are in the left lane.

\subsection{Identification of the $90 \mathrm{kDa}$ MHV-JHM 3' (+) RNA binding protein}

A large scale preparation of heparin agarose affinity matrix purified 
material was reduced, carboxymethylated and subjected to SDS-PAGE. The $90 \mathrm{kDa}$ band was excised from the gel, digested with trypsin, fractionated by HPLC and analyzed by MALDI-mass spectrometry. The three best fits with the MS data were bovine, swine, and human mitochondrial aconitases (maconitase) with $63 \%, 54 \%$, and $54 \%$ masses matched respectively. Two peptides were sequenced and yielded the sequences IVYGHLDDPANQEIER and LTIQGLK. A BLAST search of the SwissProt database revealed $100 \%$ sequence identity with residues $69-83$ and $724-$ 730 of human m-aconitase.

Proteins binding to the MHV $3^{\prime}(+) 42$ protein binding element were further purified by affinity chromatography. Ninety, 70, 58, and $40 \mathrm{kDa}$ proteins were eluted from the specific RNA affinity matrix (Fig. 2A). The $90 \mathrm{kDa}$ protein co-electrophoresed with purified bovine $\mathrm{m}$-aconitase and was immunoreactive with an anti-m-aconitase antibody (Fig. 1C). The $90 \mathrm{kDa}$ protein contained in RNA-protein complex 1 isolated by native gel electrophoresis was also recognized by this antibody (not shown). These results strongly support the identification of the $90 \mathrm{kDa}$ protein in RNAprotein complex 1 as m-aconitase.

RNase protection/gel mobility shift reactions were carried out with purified bovine mitochondrial holo- and apo-aconitase but failed to detect binding to the MHV $3^{\prime}(+) 42$ RNA. When a UV cross- linking step was added prior to electrophoresis, only the mitochondrial apo-aconitase formed a complex. This complex co-electrophoresed with complex 2 formed with cytoplasmic lysate (Fig. 2A). Specificity of binding of apo-aconitase to MHV 3'(+)42 RNA was verified by competition (Fig. 2A).

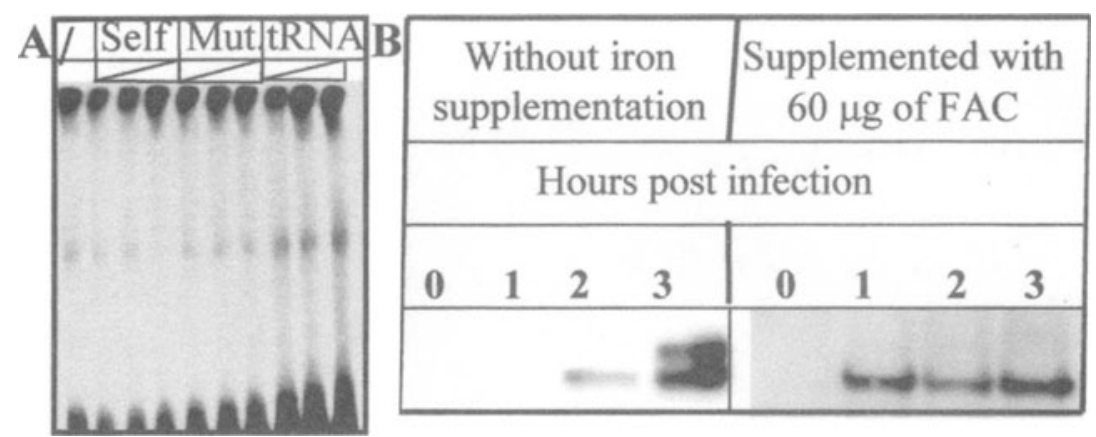

Figure 2. Panel A. Specific binding of m-apo-aconitase in the presence of competitor RNAs. B. Western Blot analysis of $\mathrm{N}$ protein levels in control and FAC treated cells. 


\subsection{Iron supplementation increases the level $\mathrm{m}$-aconitase and $\mathbf{N}$ protein}

The 5' UTR of m-aconitase contains an IRE which links cellular iron status and m-aconitase expression (Kim et al., 1996). To seek functional effects of the interaction of m-aconitase with the MHV 3'(+)42 protein binding element, we examined the effect of ferric ammonium citrate (FAC) supplementation on virus replication. The ability of MHV to form plaques was unchanged by FAC treatment of cells. Immunoblots showed that iron supplementation increased $\mathrm{N}$ protein accumulation at 1-3 h p.i. compared to untreated cultures (Fig. 2B).

\section{DISCUSSION}

Two protein binding elements have been mapped within the MHV $3(+)$ UTR and are implicated in viral replication (Liu, Yu, and Leibowitz, 1997; Yu and Leibowitz, 1995a; Yu and Leibowitz, 1995b). In this work we have demonstrated that there are at least four protein components in the RNP complex formed at the $3^{\prime}$ most cis-element in the MHV-JHM 3' UTR; maconitase is one component of the complex.

Mammalian cells have two aconitases encoded by separate nuclear genes: the mitochondrial enzyme and cytoplasmic aconitase, better known as iron regulatory protein (IRP). The IRP has 30\% amino acid identity with maconitase (Kennedy et al., 1992) and $\sim 56 \%$ overall sequence similarity. Activity of the enzymes depend on the presence of an iron sulfur [4Fe-4S] cluster in the catalytic center. IRP is a conditional cytoplasmic mRNAbinding protein which interacts with iron-responsive elements (IREs) located in the 5' UTR of ferritin mRNA and the 3' UTR of transferrin receptor (TfR) mRNA and coordinates post-transcriptional regulation of cellular iron metabolism (Klausner, Rouault, and Harford, 1993). Disassembly of the IRP iron-sulfur cluster upon iron starvation yields the RNA-binding form of the IRP. We have demonstrated that only the m-aconitase apoprotein binds to the MHV $3^{\prime}(+) 42 \mathrm{RNA}$, and that this binding is specific. M-aconitase, like its IRP homolog, is a bifunctional protein whose dynamic [4Fe-4S] cluster determines if it functions as an enzyme or as a RNA binding protein.

It is surprising that $\mathrm{m}$-aconitase, a mitochondrial matrix protein, binds to a viral RNA that replicates in the cytoplasm. RNA binding activity did not partition with the majority of $\mathrm{m}$-aconitase detected in mitochondrial lysates (not shown). We believe that m-aconitase interacts with MHV RNA prior to importation into mitochondria. Alternatively, many mitochondrial proteins 
have been demonstrated to be present outside of mitochondria under certain conditions (Soltys and Gupta, 1999).

Iron supplementation increased expression of $\mathrm{N}$ protein as early as one $\mathrm{h}$ p.i. with a corresponding change in MHV-specific mRNA levels (not shown). The effect on viral protein synthesis parallels a modest increase in $\mathrm{m}$-aconitase level in iron treated cells. We believe that $\mathrm{m}$-aconitase binding to the 3' UTR increases the expression of viral proteins, similar to the role of IRP in regulating TfR (Klausner, Rouault, and Harford, 1993).

\section{ACKNOWLEDGMENT}

This work was supported by National Multiple Sclerosis Society grant RG2203-B-6 and a gift from the Stearman family.

\section{REFERENCES}

Kennedy, M.C., Mende-Mueller, L., Blondin, G.A., and Beinert, H. (1992). Purification and characterization of cytosolic aconitase from beef liver and its relationship to the ironresponsive element binding protein. Proc Natl Acad Sci U S A 89(24), 11730-4.

Kim, H.Y., LaVaute, T., Iwai, K., Klausner, R.D., and Rouault, T.A. (1996). Identification of a conserved and functional iron-responsive element in the 5'-untranslated region of mammalian mitochondrial aconitase. J. Biol. Chem. 271, 24226-24230.

Kim, Y.-N., Jeong, Y.S., and Makino, S. (1993). Analysis of cis-acting sequences essential for coronavirus defective interfering RNA replication. Virology 197, 53-63.

Klausner, R.D., Rouault, T.A., and Harford, J.B. (1993). Regulating the fate of mRNA: the control of cellular iron metabolism. Cell 72(1), 19-28.

Leibowitz, J.L., Wilhelmsen, K.C., and Bond, C.W. (1981). The virus-specific intracellular RNA species of two murine coronaviruses: MHV-A59 and MHV-JHM. Virology 114, 3951.

Lin, Y., Liao, C., and Lai, M.M.C. (1994). Identification of the cis-acting signal for minusstrand RNA synthesis of a murine coronavirus: implications for the role of minus-strand RNA in RNA replication and transcription. J. Virol. 68(12), 8131-8140.

Lin, Y.-J., and Lai, M.M.C. (1993). Deletion mapping of a mouse hepatitis virus defective interfering RNA reveals the requirement of an internal and discontinous sequence for replication. J. Virol. 67, 6110-6118.

Liu, Q., Yu, W., and Leibowitz, J.L. (1997). A specific host cellular protein binding element near the 3' end of mouse hepatitis virus genomic RNA. Virology 232, 74-85.

Soltys, B.J., and Gupta, R.S. (1999). Mitochondrial-matrix proteins at unexpected locations: are they exported? Trends Biochem. Sci. 24, 174-177.

Yu, W., and Leibowitz, J.L. (1995a). A conserved motif at the 3' end of mouse hepatitis virus genomic RNA required for host protein binding and viral RNA replication. Virology 214, 128-138.

Yu, W., and Leibowitz, J.L. (1995b). Specific binding of host cellular proteins to multiple sites within the 3 ' end of mouse hepatitis virus genomic RNA. J. Virol. 69, 5033-5038. 